\title{
Hepatocellular Carcinoma in Liver Transplantation Candidates: Detection with Gadobenate Dimeglumine-Enhanced MRI
}

\author{
Seung Hong Choi ${ }^{1}$ \\ Jeong Min Lee ${ }^{1}$ \\ Nam C. Yu ${ }^{2}$ \\ Kyung-Suk Suh ${ }^{3}$ \\ Ja-June Jang ${ }^{4}$ \\ Se Hyung Kim ${ }^{1}$ \\ Byung Ihn Choi ${ }^{1}$
}

Keywords: gadobenate dimeglumine, hepatocellular carcinoma, liver cirrhosis, MRI

DOI:10.2214/AJR.07.2565

Received May 14, 2007; accepted after revision February 4, 2008.

'Department of Radiology and Institute of Radiation Medicine, Seoul National University College of Medicine, Clinical Research Institute, Seoul National University Hospital, 28, Yongon-dong, Chongno-gu, Seoul, 110-744, Korea. Address correspondence to J. M. Lee (leejm@ radcom.snu.ac.kr).

${ }^{2}$ Department of Radiology, David Geffen School of Medicine at the University of California, Los Angeles, Los Angeles, CA.

${ }^{3}$ Department of Surgery, Seoul National University Hospital, Seoul, Korea.

${ }^{4}$ Department of Pathology, Seoul National University Hospital, Seoul, Korea.

AJR2008; 191:529-536

0361-803X/08/1912-529

(c) American Roentgen Ray Society

OBJECTIVE. The purpose of this study was to retrospectively evaluate the diagnostic performance of dynamic gadobenate dimeglumine-enhanced MRI with explant pathologic correlation in the detection of hepatocellular carcinoma (HCC) in patients undergoing liver transplantation.

MATERIALS AND METHODS. Forty-seven patients (28 men, 19 women; mean age, 49 years) underwent dynamic gadobenate dimeglumine-enhanced MRI within 3 months before primary liver transplantation. Dynamic imaging was performed before (unenhanced) and after (hepatic arterial, portal venous, equilibrium, and 1-hour delayed phases) IV bolus administration of gadobenate dimeglumine at $0.1 \mathrm{mmol} / \mathrm{kg}$ body weight. Retrospective image analysis to detect HCC nodules was performed independently by two abdominal radiologists who had no pathologic information. On a per-nodule basis, the sensitivity and positive predictive value were calculated for the two observers. Sensitivity and specificity in the diagnosis of HCC also were evaluated. Fisher's exact test was performed to determine whether there was a detection difference between $\mathrm{HCC}$ nodules $1 \mathrm{~cm}$ in diameter or larger and nodules smaller than $1 \mathrm{~cm}$ and to evaluate the differences in causes of false-positive MRI findings based on lesion size ( $\geq 1 \mathrm{~cm}$ vs $<1 \mathrm{~cm})$.

RESULTS. Twenty-seven patients had 41 HCCs. In HCC detection, gadobenate dimeglumine-enhanced MRI had a sensitivity of $85 \%$ (35 of $41 \mathrm{HCCs}$ ) and a positive predictive value of $66 \%$ (35 of 53 readings) for observer 1 and a sensitivity of $80 \%$ (33 of 41 HCCs) and a positive predictive value of $65 \%$ (34 of 52 readings) for observer 2 . For both observers, sensitivity in the detection of HCCs $1 \mathrm{~cm}$ in diameter and larger (91-94\%) was significantly different $(p<0.05)$ from that in detection of HCCs smaller than $1 \mathrm{~cm}(29-43 \%)$. Nonneoplastic arterial hypervascular lesions more often caused false-positive diagnoses of lesions smaller than $1 \mathrm{~cm}$ in diameter $(80-86 \%)$ on MR images than of those $1 \mathrm{~cm}$ in diameter and larger $(0-25 \%)$. The difference was statistically significant $(p<0.05)$ for both observers. In diagnosis, gadobenate dimeglumine-enhanced MRI had a sensitivity of 87\% (20 of 23 patients) and a specificity of $79 \%$ (19 of 24 patients) for both observers.

CONCLUSION. Dynamic gadobenate dimeglumine-enhanced MRI has a sensitivity of $80-85 \%$ and a positive predictive value of $65-66 \%$ in the detection of HCC. The technique, however, is of limited value for detecting and characterizing lesions smaller than $1 \mathrm{~cm}$ in diameter.

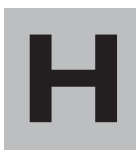
epatocellular carcinoma (HCC) accounts for more than $50 \%$ of deaths related to underlying cirrhosis [1]. Although sonography, largely because of its relatively low cost and ready accessibility, is the most widely used technique for HCC screening and surveillance [2], its heavy operator dependence and limited depiction of small $(<2 \mathrm{~cm}$ in diameter) nodules remain substantial disadvantages [3]. The latter limitation is particularly troublesome because tumor stage at diagno- sis determines patient eligibility for and the potential success of curative treatments. Therefore, CT and MRI aided by technologic advances that afford improved spatial, temporal, and contrast resolution are regarded as promising alternative surveillance tools for detecting HCC $[4,5]$. Contrast-enhanced dynamic multiphasic imaging has substantial performance benefits because most HCCs are hypervascular lesions that typically become enhanced during the hepatic arterydominant phase of imaging [6]. 


\section{Choi et al.}

A 3D gradient-echo sequence and parallel acquisition techniques facilitate dynamic contrast-enhanced imaging of HCC [7-12]. Gadobenate dimeglumine has been found useful for liver MRI. Because of its weak binding with serum albumin, this gadolinium-based contrast agent has twofold greater $\mathrm{T} 1$ relaxivity $\left(9.7 \mathrm{~L} \cdot \mathrm{mmol}^{-1} \cdot \mathrm{s}^{-1}\right.$ at $\left.0.47 \mathrm{~T}\right)$ in human plasma than do other gadolinium-based agents [8, 10, 13-19]. Gadobenate dimeglumine is a gadolinium-based paramagnetic contrast agent that combines the properties of a conventional, nonspecific, gadolinium-based agent with those of a liver-targeted agent, improving the rate of detection of focal hepatic lesions $[8,18,20]$. Because it is taken up by functioning hepatocytes and excreted in bile, use of gadobenate dimeglumine results in marked and prolonged enhancement of normal liver parenchyma for as long as 2 hours on T1-weighted images with minimal or no enhancement of nonhepatocellular tumors, improving the utility of MRI in characterizing focal liver lesions [21-23]. In addition, several studies have shown successful use of gadobenate dimeglumine in MR angiography of a variety of vascular territories and in MRI of the brain [24-26].

Previous studies of gadobenate dimeglumine-enhanced MRI have been limited by the absence of complete correlation between the pathologic and imaging findings. These studies have relied on biopsy or surgical resection specimens for evaluation of the accuracy of imaging detection and characterization of cirrhotic nodules, the resulting bias being toward positive study results. Because some dysplastic nodules [27, 28] and lesions of focal nodular hyperplasia $[29,30]$ also become enhanced during the hepatic arterial phase, the presence of arterial phase enhancement is not specific for the presence of malignancy. In addition, arterial phase enhancement can be seen with hemangiomas, arterial-portal venous shunts, and aberrant venous drainage [31, 32].

To our knowledge, no study of transplanted livers has been conducted to evaluate the diagnostic performance of dynamic gadobenate dimeglumine-enhanced MRI in the detection of HCC. The aim of this study was to perform imaging and pathologic examinations of liver explants to evaluate the diagnostic performance of dynamic gadobenate dimeglumine-enhanced MRI with a $3 \mathrm{D}$ gradient-echo sequence in the detection of HCC in patients undergoing liver transplantation.

\section{Materials and Methods Patients}

Institutional review board approval was obtained for retrospective review of patient medical records, and informed consent was waived. Between October 2003 and July 2005 at our institution, 113 patients underwent liver transplantation because of cirrhosis. For patients with HCC, the Milan criteria were used to determine eligibility for transplantation [33]. Nineteen patients were excluded because they were 18 years old or younger. Eight patients who had undergone previous liver transplantation also were excluded. Thirty-nine patients were excluded because they had undergone dynamic gadobenate dimeglumine-enhanced MRI more than 3 months before transplantation $(n=19)$ or had undergone only dynamic liver CT in a preoperative evaluation $(n=20)$. Finally, 47 consecutively enrolled patients fulfilling the following inclusion criteria composed the study population: age greater than 18 years, no previous liver transplantation, and dynamic gadobenate dimeglumine-enhanced MRI performed no more than 3 months before transplantation. Of the 47 patients, 28 were men and 19 were women. The mean age was 49 years (range, 35-62 years). The mean time of dynamic gadobenate dimeglumine-

\section{TABLE I: Patient Characteristics}

\begin{tabular}{|c|c|c|}
\hline Variable & All Patients $(n=47)$ & $\begin{array}{l}\text { Patients with Hepatocellular } \\
\text { Carcinoma }(n=23)\end{array}$ \\
\hline Mean age (y) & 49 (range, 35-62) & 50 (range, 38-62) \\
\hline Sex ratio (M/F) & $28 / 19$ & $17 / 6$ \\
\hline \multicolumn{3}{|l|}{ Cause of liver disease } \\
\hline Hepatitis B virus & 37 & 20 \\
\hline Hepatitis $\mathrm{C}$ virus & 3 & 3 \\
\hline Alcohol & 4 & 0 \\
\hline Other & 3 & 0 \\
\hline \multicolumn{3}{|l|}{ Child-Pugh class } \\
\hline$A$ & 4 & 3 \\
\hline B & 16 & 9 \\
\hline C & 27 & 11 \\
\hline \multicolumn{3}{|l|}{$\alpha$-Fetoprotein level (ng/mL) } \\
\hline$<10$ & 25 & 8 \\
\hline $10-100$ & 14 & 8 \\
\hline $101-400$ & 3 & 3 \\
\hline$>400$ & 5 & 4 \\
\hline \multicolumn{3}{|l|}{ Previous treatment } \\
\hline Transcatheter arterial chemoembolization & 7 & 7 \\
\hline Percutaneous ethanol injection & 1 & 1 \\
\hline $\begin{array}{l}\text { Transcatheter arterial chemoembolization } \\
\text { plus percutaneous ethanol injection }\end{array}$ & 1 & 1 \\
\hline
\end{tabular}

enhanced MRI before liver transplantation was 27 days (range, 3-78 days). Table 1 summarizes the patient characteristics.

Nine patients in whom HCC was suspected because of the features of hypervascular lesions on CT or MRI and elevation of $\alpha$-fetoprotein level received the following neoadjuvant treatment while awaiting a donor, but the nodules were not confirmed with histopathologic examination: transcatheter arterial chemoembolization alone in seven patients, percutaneous ethanol injection alone in one patient, and a combination of these two treatments in one patient. These nodules were excluded from our analysis. The mean interval between treatment and pretransplantation imaging was 185 days (range, 90-332 days).

\section{MRI Technique}

All MR images of the entire liver were obtained with a 1.5-T unit (Sonata, Siemens Medical Solutions) with a phased-array torso coil. The following sequences for HCC evaluation were used in all patients: transverse T2-weighted turbo spin-echo sequence without fat saturation (TR/ TE, 2,700/102; flip angle, $150^{\circ}$; echo-train length, 29; slice thickness, $6 \mathrm{~mm}$ ), transverse T2-weighted HASTE sequence (900/100; flip angle, $150^{\circ}$; 
echo-train length, 256; slice thickness, $6 \mathrm{~mm}$ ), transverse T1-weighted gradient-echo in-phase (87/5; flip angle, $70^{\circ}$; slice thickness, $6 \mathrm{~mm}$ ) and out-of-phase (87/2.4; flip angle, $70^{\circ}$; slice thickness, $6 \mathrm{~mm}$ ) sequences, and transverse dynamic gadobenate dimeglumine-enhanced T1weighted volumetric interpolated breath-hold examination (VIBE) with fat saturation (3.6/1.7; flip angle, $12^{\circ}$; slice thickness, $2.5 \mathrm{~mm}$; matrix size, $320 \times 170$ ). T1-weighted VIBE images with sensitivity-encoding with a reduction factor of 2 were acquired in a single breath-hold of 18 seconds. Sensitivity-encoding with a reduction factor of 2 was applied in an in-plane phaseencoding direction in 3D-dynamic imaging between two directions, that is, the in-plane phaseencoding direction and the partition direction.

Dynamic imaging was performed before (unenhanced) and after (hepatic arterial, portal venous, equilibrium, and 1-hour delayed phases) IV bolus administration of gadobenate dimeglumine $(0.1$ $\mathrm{mmol} / \mathrm{kg}$ body weight at $2-2.5 \mathrm{~mL} / \mathrm{s}$ ) through a 20 to 22-gauge antecubital angiographic venous catheter with a power injector (Stellant Dual, Siemens Medical Solutions) followed by a $10-\mathrm{mL}$ saline flush. The average volume of contrast material was $13.2 \mathrm{~mL}$ (range, 10.4-15.8 mL). Scanning delay times were determined with real-time MRI fluoroscopic monitoring after contrast administration. Acquisition of hepatic arterial phase images was started manually at enhancement of the distal thoracic aorta. The mean scanning delay for the arterial phase was approximately 25 seconds (range, 20-30 seconds). Portal venous phase and equilibrium phase imaging was performed for 60 and 180 seconds, respectively, after injection of contrast medium. One-hour delayed phase images were obtained with the VIBE sequence.

\section{Image Analysis}

Retrospective image analysis was performed independently by two abdominal imagers ( 15 and 8 years of radiology experience). The readers were informed that the patients had undergone liver transplantation and had explant pathologic correlation but were blinded to the pathologic results. All MR images were reviewed on a PACS workstation (Marosis, Marotech). The cases were randomly listed on a PACS by one observer. Each observer recorded the number and sizes of the focal lesions, and all lesions were recorded on a liver map. Lesion size was estimated on transverse MR images by measurement of the maximum diameter with an electronic ruler.

To promote objectivity in image interpretation, criteria for HCC were provided to the two radiologists. Nodules exhibiting enhancement during the hepatic arterial phase and lacking portal venous supply during the portal venous or equilibrium phase were regarded as HCC nodules [4 34-36]. Nodules exhibiting enhancement during the hepatic arterial phase and hypointensity on the 1-hour delayed phase images were also regarded as HCC [37]. A hypointense lesion on dynamic sequences and delayed images with a signal intensity higher than that of adjacent liver parenchyma but lower than that of CSF or gallbladder on T2-weighted turbo spin-echo sequences also was regarded as HCC [38, 39]. In addition, a nodule with hyperintensity on T1weighted images and with isointensity or hypointensity during both phases of dynamic study or isointensity or hypointensity on T2-weighted images was regarded as a dysplastic nodule [40] Nodules that appeared only during the hepatic arterial phase and that had no pathologic correlate were defined as nonneoplastic arterial hypervascular lesions.

In cases of false-negative or false-positive findings on gadobenate dimeglumine-enhanced MR images, the images were reanalyzed after retrospective review by the same observers to determine the causes of the misinterpretations with regard to the size of the HCC nodule and lesion size as measured on MR images. In falsepositive cases, lesions that became enhanced on arterial phase MR images without corresponding pathologic findings, including HCC, dysplastic nodule, and regenerative nodule, were regarded as nonneoplastic arterial hypervascular lesions.

\section{Reference Standard}

As a preoperative evaluation, all imaging studies, including CT and MR images, were interpreted for HCC burden by consensus of the same two gastrointestinal radiologists before liver transplantation, and the pathologist was notified by written report regarding all suspected $\mathrm{HCC}$ lesions. The sonographic information was transferred to the two radiologists for tumor burden evaluation.

Gross and histologic analyses of all explanted livers were performed by a hepatobiliary pathologist with 25 years of experience. The presence or absence of all lesions identified at preoperative interpretation of the images was determined histologically on a lesion-by-lesion basis. In addition, all other visible nodules on the gross specimens that were distinct from the surrounding liver tissue also were evaluated histologically. All explanted livers were initially sectioned at 5-mm or thinner intervals in the sagittal plane. If an imaged lesion was not found in the explant, representative histologic sections were obtained from the region of the liver that best corresponded to the lesion location at imaging. For patients with no lesions detected at imaging, the pathologic specimens were carefully reviewed for the presence of HCC. The liver slices were photographed, and all lesions other than regenerative nodules were sampled for histologic examination. According to the diagnostic criteria of the International Working Party [41], for nodular hepatocellular lesions, the routinely $\mathrm{H}$ and $\mathrm{E}-$-stained slices from the nodules were classified as follows: regenerative nodule; dysplastic nodule, low grade; dysplastic nodule, high grade; small HCC $(<2$ $\mathrm{cm})$; or $\mathrm{HCC}$ (> $2 \mathrm{~cm})$.

In most cases, a radiologist was not present during sectioning of the explanted livers. Discrepancy was minimized by active communication between the radiologists and the pathologist regarding the multiplanar reconstruction presentation of the axial images to guide explant sectioning.

\section{Statistical Analysis}

Fisher's exact test was performed to determine whether there was a difference in the numbers of patients in the included and excluded populations in this study. Interobserver variability for detection of hepatic nodules was evaluated with nonweighted binary kappa statistics. A kappa value of 0.01-0.20 was classified as minor agreement; 0.21-0.40, fair; 0.41-0.60, moderate; 0.61-0.80, high; and $0.81-1.00$, excellent. To evaluate HCC burden, the respective sensitivities and positive predictive values for the two observers were calculated. The sensitivity of HCC detection for lesions larger than $2 \mathrm{~cm}, 1-2 \mathrm{~cm}$, and less than $1 \mathrm{~cm}$ in diameter and for all lesions was calculated on a per-lesion basis. Sensitivity and specificity for the diagnosis of HCC also were evaluated. Fisher's exact test was performed to determine whether there was a detection difference between HCCs $1 \mathrm{~cm}$ or larger and those smaller than $1 \mathrm{~cm}$ in diameter. Fisher's exact test also was used to evaluate the differences in causes of false-positive results according to lesion size measured on MR images ( $\geq 1 \mathrm{~cm}$ vs $<1$ $\mathrm{cm})$. A value of $p<0.05$ was considered to indicate a statistically significant difference. Statistical analyses were performed with the MedCalc program (version 9.1.0.1, MedCalc Software).

\section{Results \\ Pathologic Findings}

Twenty-three of the 47 patients in the study had 41 HCC nodules (10 patients had one lesion; nine, two lesions; three, three lesions; and one, four lesions). The mean nodule diameter was $2.2 \mathrm{~cm}$ (range, $0.3-6.5 \mathrm{~cm}$ ). HCC differentiation was good in four cases, moderate in 36 cases, and poor in one case (Table 2). A total of 30 non-HCC nodules were identified and consisted of 26 dysplastic nodules (mean diameter, $0.9 \mathrm{~cm}$; range, $0.5-1.7 \mathrm{~cm}$ ), 


\section{Choi et al.}

TABLE 2: Size and Differentiation of Hepatocellular Carcinoma Nodules $(n=4 I)$

\begin{tabular}{l|c}
\hline \multicolumn{1}{c|}{ Size and Differentiation } & No. \\
\hline$>2 \mathrm{~cm}$ & 16 \\
Well differentiated & $\mathrm{NA}$ \\
Moderately differentiated & 16 \\
Poorly differentiated & $\mathrm{NA}$ \\
$1-2 \mathrm{~cm}$ & 18 \\
Well differentiated & 3 \\
Moderately differentiated & 14 \\
Poorly differentiated & 1 \\
$<1 \mathrm{~cm}$ & 7 \\
Well differentiated & 1 \\
Moderately differentiated & 6 \\
Poorly differentiated & $\mathrm{NA}$ \\
\hline Total & 41 \\
\hline
\end{tabular}

Note-NA = not available.

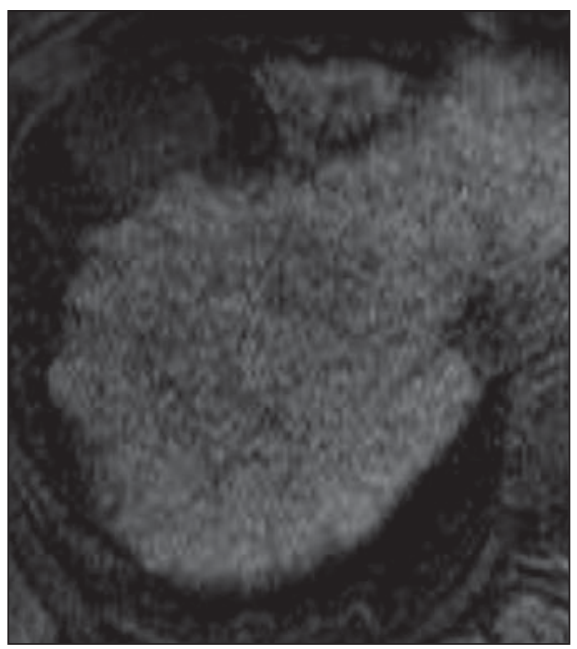

A

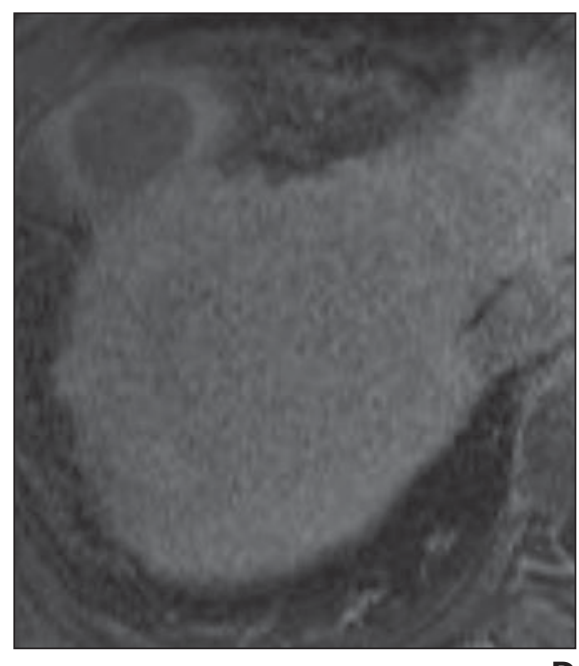

two regenerative nodules $(0.5$ and $0.8 \mathrm{~cm})$, one biliary adenoma $(0.6 \mathrm{~cm})$, and one hemangioma $(2.2 \mathrm{~cm})$, which had typical findings on MR images.

Forty-seven of the 66 patients excluded from this study were older than 18 years. Twelve of those patients had 20 HCC nod- ules: seven had one lesion; three, two lesions; one patient, three lesions; and one, four lesions. The mean diameter of these nodules was $2.5 \mathrm{~cm}$ (range, $0.8-5.3 \mathrm{~cm}$ ). The excluded group had a lower prevalence of HCC than did the patients included in the study $(p=0.032)$.

TABLE 3: Sensitivity and Positive Predictive Value in Detection of Hepatocellular Carcinoma with Gadobenate Dimeglumine-Enhanced MRI

\begin{tabular}{l|c|c}
\hline \multicolumn{1}{c|}{ Characteristic } & Observer 1 & Observer 2 \\
\hline Sensitivity & & \\
Total & $85(35 / 41)$ & $80(33 / 41)$ \\
$>2 \mathrm{~cm}$ & $100(16 / 16)$ & $100(16 / 16)$ \\
$1-2 \mathrm{~cm}$ & $89(16 / 18)$ & $83(15 / 18)$ \\
$\geq 1 \mathrm{~cm}$ & $94(32 / 34)$ & $91(31 / 34)$ \\
$<1 \mathrm{~cm}$ & $43(3 / 7)$ & $29(2 / 7)$ \\
Positive predictive value & $66(35 / 53)$ & $65(34 / 52)$ \\
\hline
\end{tabular}

Note-Values are percentages with corresponding counts in parentheses.

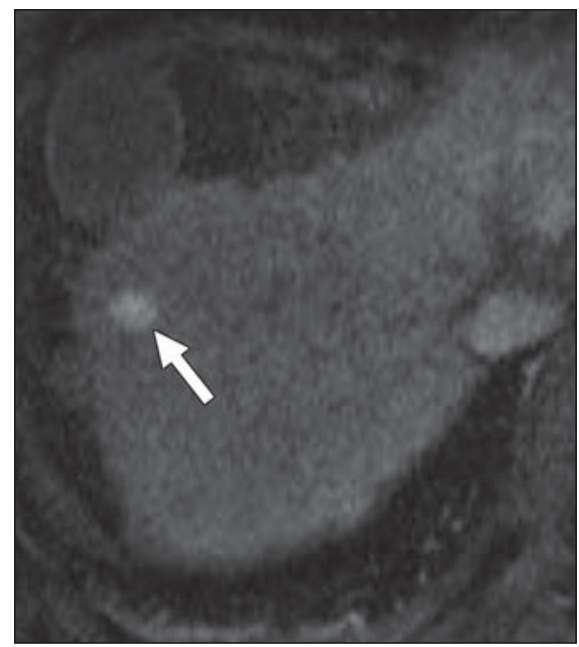

B

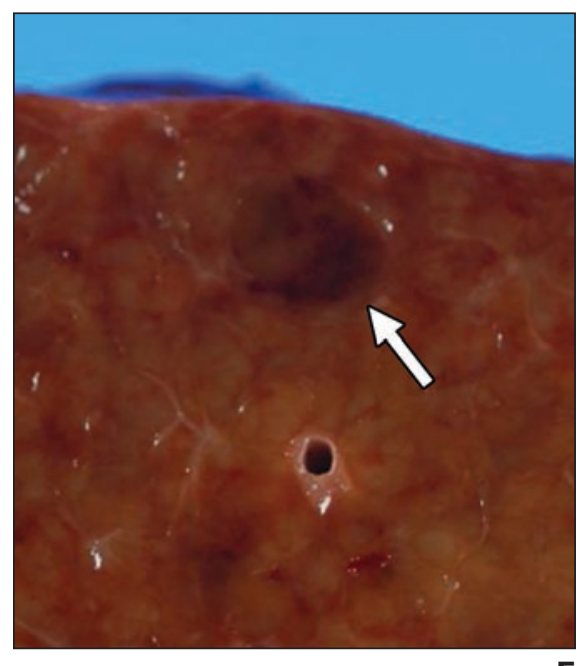

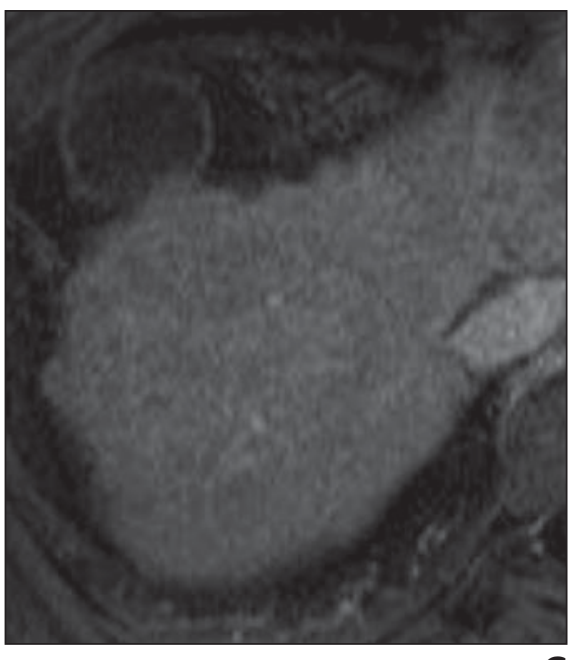

C

Fig. 1-55-year-old man with macronodular cirrhosis and $1.3-\mathrm{cm}$-diameter hepatocellular carcinoma in liver segment 7.

A, Unenhanced axial T1-weighted volumetric interpolated breath-hold examination (VIBE) image (TR/TE, 3.6/1.7; flip angle, $12^{\circ}$ ) shows no liver lesion. B, Hepatic arterial phase gadobenate dimeglumineenhanced axial T1-weighted VIBE image (3.6/1.7; flip angle, $12^{\circ}$ ) shows enhancing nodule (arrow) in liver segment 7.

C. Portal venous phase gadobenate dimeglumineenhanced axial T1-weighted VIBE image (3.6/1.7; flip angle, $12^{\circ}$ ) shows no liver lesion.

D, Delayed axial T1-weighted VIBE image (3.6/1.7 flip angle, $12^{\circ}$ ) obtained 1 hour after gadobenate dimeglumine administration shows no liver lesion. E, Photomicrograph of sagittal section of pathologic specimen shows 1.3-cm-diameter hepatocellular carcinoma nodule (arrow) in segment 7. 


\section{Image Analysis}

Interobserver variability between the two readers suggested excellent agreement $(\kappa=$ $0.815 \pm 0.093[\mathrm{SD}])$ for detection of hepatic nodules. Table 3 shows that for HCC detection, observer 1 had a sensitivity of $85 \%$ (35 of 41 HCCs) and a positive predictive value of $66 \%$ (35 of 53 readings). Observer 2 had a sensitivity of $80 \%$ (33 of $41 \mathrm{HCCs}$ ) and a positive predictive value of $65 \%$ (34 of 52 readings).

In terms of sensitivity, the two observers had significant differences ( $p=0.004, p=$ 0.001 ) between detection of HCCs $1 \mathrm{~cm}$ or larger (94\% and $91 \%$ ) and those smaller than

TABLE 4: False-Positive Findings in Detection of Hepatocellular Carcinoma on Gadobenate Dimeglumine-Enhanced MRI

\begin{tabular}{l|c|c}
\hline \multicolumn{1}{c|}{ Finding } & Observer 1 & Observer 2 \\
\hline $1-2 \mathrm{~cm}$ & 3 & 3 \\
Dysplastic nodule & NA & 1 \\
$\begin{array}{c}\text { Nonneoplastic arterial } \\
\text { hypervascular lesion }\end{array}$ & & \\
$<1 \mathrm{~cm}$ & & \\
Dysplastic nodule & 2 & 2 \\
Nonneoplastic arterial \\
hypervascular lesion & 12 & 12 \\
Regenerative nodule & 1 & 0 \\
\hline Total & 18 & 18 \\
\hline
\end{tabular}

Note-NA = not available.

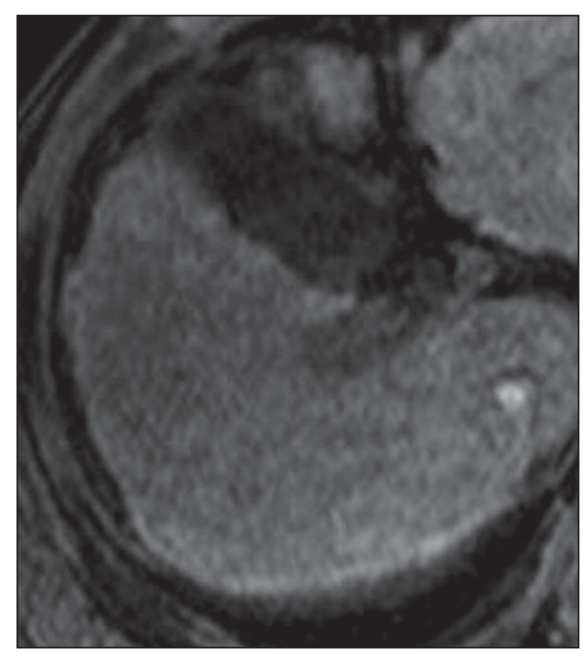

$\mathbf{A}$
$1 \mathrm{~cm} \mathrm{(43 \%} \mathrm{and} \mathrm{29 \% )} \mathrm{(Fig.} \mathrm{1).} \mathrm{The} \mathrm{two} \mathrm{and}$ three HCC nodules $1 \mathrm{~cm}$ or larger missed by observers 1 and 2 on gadobenate dimeglumine-enhanced MR images were pathologically confirmed to be well-differentiated HCC (Table 3).

All MRI lesions measuring more than $2 \mathrm{~cm}$ in diameter were determined to be HCC. Table 4 summarizes the causes of all false-positive diagnoses of lesions measuring $2 \mathrm{~cm}$ in diameter or smaller on MRI. Nonneoplastic arterial hypervascular lesions more often caused false-positive MRI diagnoses of lesions measuring less than $1 \mathrm{~cm}$ in diameter (80\%, 12 of 15 readings; $86 \%, 12$ of 14 readings) than of lesions $1 \mathrm{~cm}$ in diameter or larger $(0 \%$, none of three readings; $25 \%$, one of four readings) with statistically significant differences ( $p=0.025, p=0.044)$ for the two observers (Figs. 2 and 3 ). For both observers, the false-positive MRI lesions measuring $1 \mathrm{~cm}$ in diameter or greater were dysplastic nodules; three high-grade dysplastic nodules $1 \mathrm{~cm}$ in diameter or larger were interpreted as HCC nodules by both observers. Both observers' readings indicated that the sensitivity for the diagnosis of HCC was $87 \%$ (20 of 23 patients) and the specificity was $79 \%$ (19 of 24).

\section{Discussion}

Our study results show that gadobenate dimeglumine-enhanced MRI had a sensitivity of $80-85 \%$ and positive predictive value of $65-66 \%$ in the detection of HCC nodules

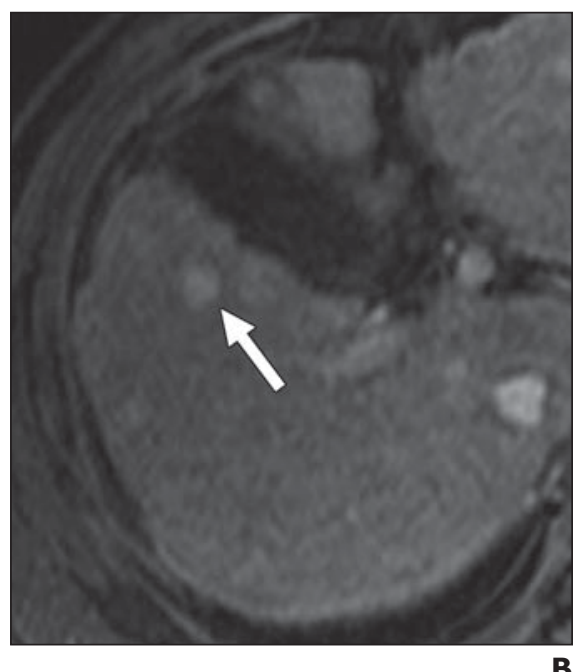

when used together with explant pathologic correlation. In addition, gadobenate dimeglumine-enhanced MRI had a higher sensitivity for detection of HCC nodules $1 \mathrm{~cm}$ in diameter or larger (91-94\%) than for those smaller than $1 \mathrm{~cm}$ in diameter (29-43\%), a significant difference $(p<0.05)$. The performance gain with gadobenate dimeglumine-enhanced MRI was excellent for nodules measuring $1 \mathrm{~cm}$ or more in diameter. We attribute this MRI performance to use of a modified 3D gradientecho technique and the almost twofold greater T1 relaxivity of gadobenate dimeglumine relative to conventional gadolinium-based agents and to the excellent intrinsic soft-tissue contrast enhancement of MRI [8, 18, 20, 42-45].

The fat-suppressed 3D gradient-echo MR sequence (VIBE) we used for dynamic MRI allowed shorter scan times and use of thinner (1-3-mm) sections without image degradation through use of asymmetric k-space sampling and interpolation [42-45]. In our study, however, gadobenate dimeglumine-enhanced MRI had only limited utility in the detection of $\mathrm{HCC}$ nodules smaller than $1 \mathrm{~cm}$ in diameter and well-differentiated lesions, which often appear hypovascular because of insufficient arterial neovascularization in the setting of a decreased portal supply. A previous study [46] showed relatively high sensitivity (82$88 \%$ ) in the detection of HCC nodules $1 \mathrm{~cm}$ in diameter or smaller with gadobenate dimeglumine-enhanced MRI compared with our finding (29-43\% sensitivity). However, the

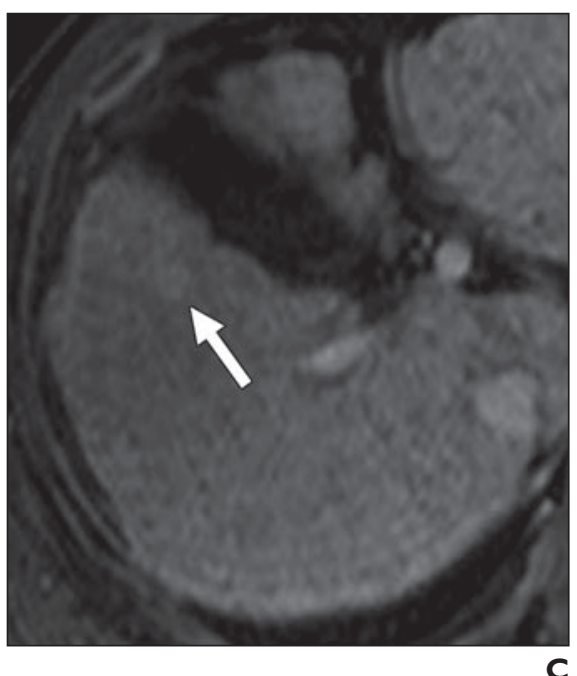

Fig. 2-47-year-old man with macronodular cirrhosis.

A, Unenhanced axial T1-weighted volumetric interpolated breath-hold examination (VIBE) image (TR/TE, 3.6/1.7; flip angle, $12^{\circ}$ ) shows no liver lesion.

B, Hepatic artery phase gadobenate dimeglumine-enhanced axial T1-weighted VIBE image (3.6/1.7; flip angle, $\left.12^{\circ}\right)$ shows enhancing 9-mm-diameter lesion (arrow) in liver segment 5. No nodule was identified at pathologic examination. Lesion was considered nonneoplastic arterial hypervascular lesion.

C, Portal venous phase gadobenate dimeglumine-enhanced axial T1-weighted VIBE image $\left(3.6 / 1.7 ;\right.$ flip angle, $\left.12^{\circ}\right)$ shows subtle enhancing lesion (arrow) in liver segment 5 . 

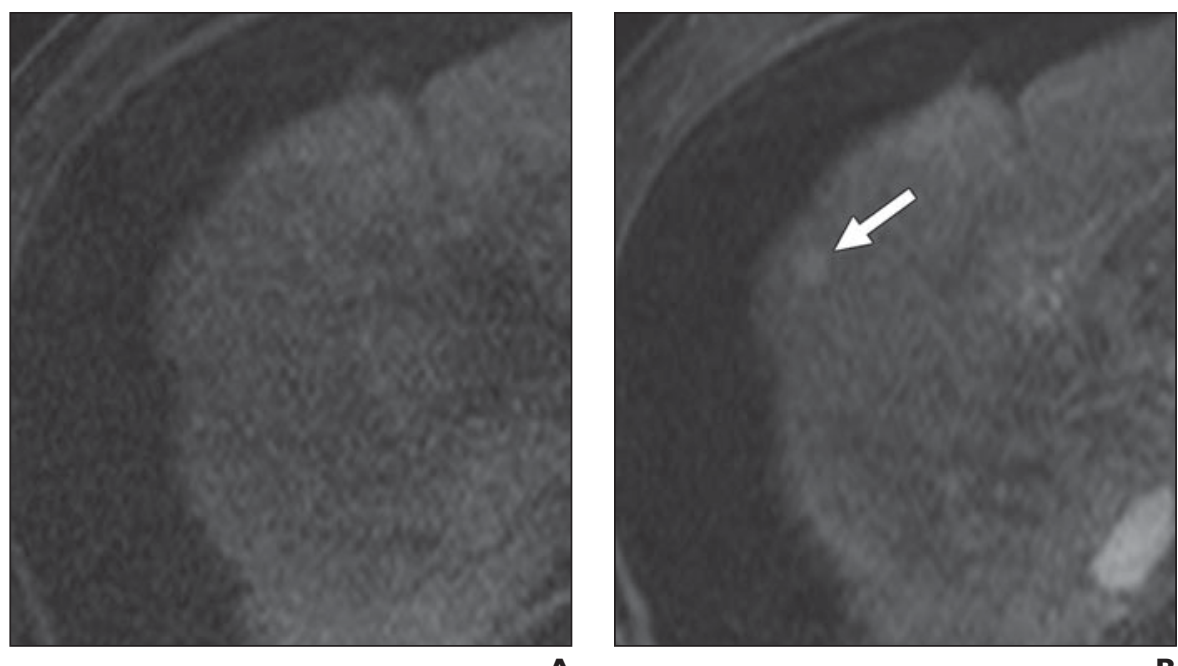

A
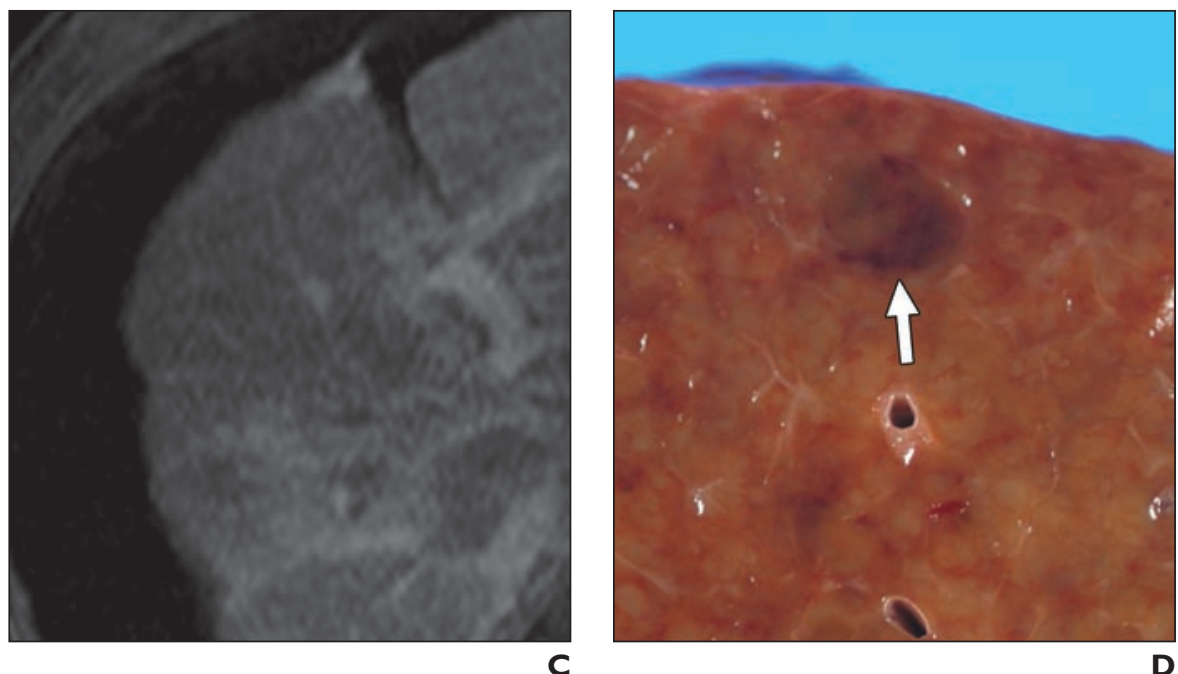

Fig. 3-38-year-old man with macronodular cirrhosis and 8-mm-diameter dysplastic nodule in liver segment 4. A, Unenhanced axial T1-weighted volumetric interpolated breath-hold examination (VIBE) image (TR/TE, 3.6/1.7; flip angle, $12^{\circ}$ ) shows no liver lesion.

B, Hepatic arterial phase gadobenate dimeglumine-enhanced axial T1-weighted VIBE image (3.6/1.7; flip angle, $12^{\circ}$ ) shows enhancing mass (arrow) in liver segment 4.

C. Portal venous phase gadobenate dimeglumine-enhanced axial T1-weighted VIBE image (3.6/1.7; flip angle,

$12^{\circ}$ ) shows no liver lesion.

D, Photomicrograph of sagittal section of pathologic specimen shows 8-mm-diameter dysplastic nodule (arrow) in segment 4.

reference standard in that study was limited liver resection, image-guided biopsy, or iodized oil CT combined with elevated $\alpha$-fetoprotein level, and such an approach might hide observer bias and tend to lead to underestimation of extent of disease [4].

In terms of positive predictive value, our study showed lower performance $(65-66 \%)$ than previous studies $(85-96 \%)$ [8, 46]. It has been reported [8] that the combination of the higher enhancing capability of gadobenate dimeglumine and the VIBE sequence tends to maximize sensitivity in detection of enhancing hepatic lesions regardless of the presence of an arterioportal shunt or of a true hypervascular liver lesion. Therefore, in our study, the advanced cirrhotic state of the livers was associated with foci of nonmalignant hypervascularity, including nonneoplastic arterial hypervascular lesions and enhancing dysplastic or regenerative nodules, thereby accounting for the false-positive findings $[32,36]$. In addition in our study, the major causes of false-positive diagnoses of lesions of smaller than $1 \mathrm{~cm}$ on MRI were different from those for lesions $1 \mathrm{~cm}$ in diameter or larger. Nonneoplastic arterial hypervascular lesions were the major cause of false-positive diagnoses of lesions smaller than $1 \mathrm{~cm}$ in diameter (80-86\%) and dysplastic nodules the cause in the case of lesions $1 \mathrm{~cm}$ in diameter or larger (85-100\%). The results of this study partially correlate with those of a study by Holland et al. [47], who reported that $60 \%$ of nonneoplastic arterial hypervascular lesions were smaller than $1 \mathrm{~cm}$ in diameter.

In our study, the quality of delayed phase images was poor owing to weak enhancement of hepatic parenchyma related to poor hepatocyte function in advanced cirrhosis. Therefore, the diagnostic value of delayed phase imaging in characterization and detection of hepatocellular nodules in cirrhotic liver was limited. These findings are similar to those in a study by Grazioli et al. [21], in which positive correlation (Spearman's correlation, 0.359) was observed between the degree of liver failure and worsening of lesion-to-liver contrast-to-noise ratio on delayed phase images.

At our institution, patients waiting for liver transplantation usually underwent followup with dynamic liver CT, and gadobenate dimeglumine-enhanced MRI was used for those who had suspicious nodules on CT images or could not undergo dynamic liver CT because of a medial problem. The 47 adult patients excluded from this study did not undergo gadobenate dimeglumine-enhanced MRI because of definite hypervascular lesions on CT images and an increased $\alpha$-fetoprotein level in some patients and no abnormality on CT in others. Thus only 12 patients had histopathologic proof of HCC, and four of these patients were reported to have no lesions suspected of being HCC on CT. Finally, the main population of this study consisted of patients with suspicious nodules on dynamic liver CT, which might have led to selection bias that increased the sensitivity of HCC detection.

A radiologist was not present at the time of liver explant sectioning, and the reference standard was the written report by the pathologist because this study was performed retrospectively. At our institution, however, liver specimens are evaluated under strict protocol, which is reflected by the relatively low sensitivity for HCC nodules smaller than $1 \mathrm{~cm}$ in this study compared with previous studies.

Apart from the intrinsic limits of any retrospective study, our study had several limitations. First, that the imaging plane (transverse) differed from the pathologic plane (sagittal) potentially complicated lesion 


\section{MRI of Hepatocellular Carcinoma}

matching and localization. Although we made an effort to match the in vivo MR images with the whole explanted livers, precise lesion-by-lesion correlation was extremely difficult because of the difference between the pathologic and imaging planes. Second, many patients included in our study had advanced cirrhosis, which might have decreased the detection and characterization performance of gadobenate dimeglumineenhanced MRI for focal liver lesions.

Our study results show that dynamic gadobenate dimeglumine-enhanced MRI has a sensitivity of $80-85 \%$ and positive predictive value of $65-66 \%$ in the detection of HCC nodules with explant pathologic correlation. For lesions smaller than $1 \mathrm{~cm}$ in diameter, however, this technique has limitations for detection and characterization, accounting for most of our false-negative and false-positive findings.

\section{References}

1. Murakami T, Hori M, Kim T, Kawata S, Abe H, Nakamura H. Multidetector row CT and MR imaging in diagnosing hepatocellular carcinoma. Intervirology 2004; 47:209-226

2. Chalasani N, Said A, Ness R, Hoen H, Lumeng L. Screening for hepatocellular carcinoma in patients with cirrhosis in the United States: results of a national survey. Am J Gastroenterol 1999; 94:2224-2229

3. Bennett GL, Krinsky GA, Abitbol RJ, Kim SY, Theise ND, Teperman LW. Sonographic detection of hepatocellular carcinoma and dysplastic nodules in cirrhosis: correlation of pretransplantation sonography and liver explant pathology in $200 \mathrm{pa}-$ tients. AJR 2002; 179:75-80

4. Burrel M, Llovet JM, Ayuso C, et al. MRI angiography is superior to helical CT for detection of HCC prior to liver transplantation: an explant correlation. Hepatology 2003; 38:1034-1042

5. Van Thiel DH, Yong S, Li SD, Kennedy M, Brems J. The development of de novo hepatocellular carcinoma in patients on a liver transplant list: frequency, size, and assessment of current screening methods. Liver Transpl 2004; 10:631-637

6. Yamashita Y, Mitsuzaki K, Yi T, et al. Small hepatocellular carcinoma in patients with chronic liver damage: prospective comparison of detection with dynamic MR imaging and helical CT of the whole liver. Radiology 1996; 200:79-84

7. Pauleit D, Textor J, Bachmann R, at al. Hepatocellular carcinoma: detection with gadolinium- and ferumoxides-enhanced MR imaging of the liver. Radiology 2002; 222:73-80

8. Kim YK, Kim CS, Lee YH, Kwak HS, Lee JM. Comparison of superparamagnetic iron oxide- enhanced and gadobenate dimeglumine-enhanced dynamic MRI for detection of small hepatocellular carcinomas. AJR 2004; 182:1217-1223

9. Ito K, Fujita T, Shimizu A, et al. Multiarterial phase dynamic MRI of small early enhancing hepatic lesions in cirrhosis or chronic hepatitis: differentiating between hypervascular hepatocellular carcinomas and pseudolesions. AJR 2004; 183:699-705

10. Mori K, Yoshioka H, Takahashi N, et al. Triple arterial phase dynamic MRI with sensitivity encoding for hypervascular hepatocellular carcinoma: comparison of the diagnostic accuracy among the early, middle, late, and whole triple arterial phase imaging. AJR 2005; 184:63-69

11. Yoshioka H, Takahashi N, Yamaguchi M, Lou D, Saida Y, Itai Y. Double arterial phase dynamic MRI with sensitivity encoding (SENSE) for hypervascular hepatocellular carcinomas. J Magn Reson Imaging 2002; 16:259-266

12. Takahashi N, Yoshioka H, Yamaguchi M, Saida Y, Itai Y. Accelerated dynamic MR imaging with a parallel imaging technique for hypervascular hepatocellular carcinomas: usefulness of a test bolus in examination and subtraction imaging. $J$ Magn Reson Imaging 2003; 18:80-89

13. Pirovano G, Vanzulli A, Marti-Bonmati L, et al. Evaluation of the accuracy of gadobenate dimeglumine-enhanced MR imaging in the detection and characterization of focal liver lesions. AJR 2000; 175:1111-1120

14. Petersein J, Spinazzi A, Giovagnoni A, et al. Focal liver lesions: evaluation of the efficacy of gadobenate dimeglumine in MR imaging: a multicenter phase III clinical study. Radiology 2000 215:727-736

15. Caudana R, Morana G, Pirovano GP, et al. Focal malignant hepatic lesions: MR imaging enhanced with gadolinium benzyloxypropionictetra-acetate (BOPTA): preliminary results of phase II clinical application. Radiology 1996; 199:513-520

16. Schneider G, Maas R, Schultze Kool L, et al. Low-dose gadobenate dimeglumine versus standard dose gadopentetate dimeglumine for contrast-enhanced magnetic resonance imaging of liver: an intra-individual crossover comparison. Invest Radiol 2003; 38:85-94

17. Vogl TJ, Stupavsky A, Pegios W, et al. Hepatocellular carcinoma: evaluation with dynamic and static gadobenate dimeglumine-enhanced MR imaging and histopathologic correlation. Radiology 1997; 205:721-728

18. Kirchin M, Pirovano G, Spinazzi A. Gadobenate dimeglumine (Gd-BOPTA): an overview. Invest Radiol 1998; 33:798-809

19. Spinazzi A, Lorusso V, Pirovano G, Kirchin MA. Safety, tolerance, biodistribution and MR imaging enhancement of the liver with gadobenate di- meglumine. Acad Radiol 1999; 6:282-291

20. Vogl TJ, Pegios W, McMahon C, et al. Gadobenate dimeglumine: a new contrast agent for MR imaging - preliminary evaluation in healthy volunteers. AJR 1992; 158:887-892

21. Grazioli L, Morana G, Caudana R, et al. Hepatocellular carcinoma: correlation between gadobenate dimeglumine-enhanced magnetic resonance (MR) imaging and pathological findings. Invest Radiol 2000; 35:25-34

22. Kim YK, Lee JM, Kim CS. Gadobenate dimeglumine-enhanced liver MR Imaging: value of dynamic and delayed imaging for the characterization and detection of focal liver lesions. Eur Radiol 2004; 14:5-13

23. Morana G, Grazioli L, Schneider G, et al. Hypervascular hepatic lesions: dynamic and late enhancement pattern with Gd-BOPTA. Acad Radiol 2002; 9:S476-S479

24. Soulez G, Pasowicz M, Benea G, et al. Renal artery stenosis evaluation: diagnostic performance of gadobenate dimeglumine-enhanced MR angiography-comparison with DAS. Radiology 2008; 247:273-285

25. An SK, Lee JM, Suh KS, et al. Gadobenate dimeglumine-enhanced liver MRI as the sole preoperative imaging technique: a prospective study of living liver donors. AJR 2006; 187:1223-1233

26. Kreitner KF, Kunz RP, Herber S, Martenstein S, Dorweiler B, Dueber C. MR angiography of the pedal arteries with gadobenate dimeglumine, a contrast agent with increased relaxivity, and comparison with selective intraarterial DSA. J Magn Reson Imaging 2008; 27:78-85

27. Lim JH, Cho JM, Kim EY, Park CK. Dysplastic nodules in liver cirrhosis: evaluation of hemodynamics with CT during arterial portography and CT hepatic arteriography. Radiology 2000; 214:869-874

28. Efremidis SC, Hytiroglou P. The multistep process of hepatocarcinogenesis in cirrhosis with imaging correlation. Eur Radiol 2002; 12:753-764

29. Libbrecht L, Bielen D, Verslype C, et al. Focal lesions in cirrhotic explant livers: pathological evaluation and accuracy of pretransplantation imaging examinations. Liver Transpl 2002; 8:749-761

30. Quaglia A, Tibballs J, Grasso A, et al. Focal nodular hyperplasia-like areas in cirrhosis. Histopathology 2003; 42:14-21

31. Kanematsu M, Kondo H, Semelka RC, et al. Early-enhancing non-neoplastic lesions on gadolinium-enhanced MRI of the liver. Clin Radiol 2003; 58:778-786

32. Yu JS, Kim KW, Jeong MG, Lee JT, Yoo HS. Nontumorous hepatic arterial-portal venous shunts: MR imaging findings. Radiology 2000; 217:750-756

33. Mazzaferro V, Regalia E, Doci R, et al. Liver 


\section{Choi et al.}

transplantation for the treatment of small hepatocellular carcinomas in patients with cirrhosis. $N$ Engl J Med 1996; 334:693-699

34. Choi BI, Takayasu K, Han MC. Small hepatocellular carcinomas and associated nodular lesions of the liver: pathology, pathogenesis, and imaging findings. AJR 1993; 160:1177-1187

35. Krinsky GA, Lee VS, Theise ND, et al. Hepatocellular carcinoma and dysplastic nodules in patients with cirrhosis: prospective diagnosis with MR imaging and explant correlation. Radiology 2001; 219:445-454

36. Matsui O, Kadoya M, Kameyama T, et al. Benign and malignant nodules in cirrhotic livers: distinction based on blood supply. Radiology 1991; 178:493-497

37. Manfredi R, Maresca G, Baron RL, et al. Delayed MR imaging of hepatocellular carcinoma enhanced by gadobenate dimeglumine (Gd-BOPTA). J Magn Reson Imaging 1999; 9:704-710

38. Kelekis NL, Semelka RC, Worawattanakul S, et al. Hepatocellular carcinoma in North America: a multiinstitutional study of appearance on T1weighted, T2-weighted, and serial gadoliniumenhanced gradient-echo images. AJR 1998; 170:1005-1013

39. Hecht EM, Holland AE, Israel GM, et al. Hepatocellular carcinoma in cirrhotic liver: gadoliniumenhanced 3D T1-weighted MR imaging as a stand-alone sequence for diagnosis. Radiology 2006; 239:438-447

40. Earls JP, Theise ND, Weinreb JC, et al. Dysplastic nodules and hepatocellular carcinoma: thin-section MR imaging of explanted cirrhotic livers with pathologic correlation. Radiology 1996; 201:207-214

41. [No authors listed]. Terminology of nodular hepatocellular lesions: International Working Party. Hepatology 1995; 22:983-993

42. Rofsky NM, Lee VS, Laub G, et al. Abdominal MR imaging with a volumetric interpolated breath-hold examination. Radiology 1999; 212: 876-884

43. Lee VS, Lavelle MT, Rofsky NM, et al. Hepatic
MR imaging with a dynamic contrast-enhanced isotropic volumetric interpolated breath-hold examination: feasibility, reproducibility, and technical quality. Radiology 2000; 215:365-372

44. Keogan MT, Edelman RR. Technologic advances in abdominal MR imaging. Radiology 2001; 220:310-320

45. Kataoka M, Ueda H, Koyama T, et al. Contrastenhanced volumetric interpolated breath-hold examination compared with spin-echo T1-weighted imaging of head and neck tumors. AJR 2005; 184:313-319

46. Kim YK, Kim CS, Chung GH, et al. Comparison of gadobenate dimeglumine-enhanced dynamic MRI and 16-MDCT for the detection of hepatocellular carcinoma. AJR 2006; 186:149-157

47. Holland AE, Hecht EM, Hahn WY, et al. Importance of small ( $\leq 20-\mathrm{mm})$ enhancing lesions seen only during the hepatic arterial phase at MR imaging of the cirrhotic liver: evaluation and comparison with whole explanted liver. Radiology 2005; 237:938-944 